\title{
SEARCH FOR DIMENSIONAL EFFECTS IN SPIN-GLASS TRANSITION IN THIN $\mathrm{Cd}_{1-x} \mathrm{Mn}_{x}$ Te MULTILAYERS*
}

\author{
M. SaWicki, T. Dietl, T. Skośkiewicz, G. Karczewski, T. WoJtowicz \\ AND J. KossuT
}

Institute of Physics, Polish Academy of Sciences

Al. Lotników 32/46, 02-668 Warszawa, Poland

SQUID magnetic measurements of CdTe/Cd ${ }_{1-x} \mathrm{Mn}_{x} \mathrm{Te}, x \simeq 0.5$, multilayers with different CdMnTe layer thickness $w$ reveal the persistence of the spin-glass irreversibilities down to $\simeq 16 \AA$ thin layers, i.e., well beyond the previously postulated $40 \AA$ as a quasi-2D threshold. The freezing temperature $T_{f}$ is found to be a monotonic function of $w$, and it obeys a scaling law $\mathrm{T}_{\mathrm{f}} \propto w^{a}$, with $a \simeq 0.8$ similar to that for canonical spin-glasses. Magnetic properties of all studied structures are found to be independent of the orientation of the magnetic field.

PACS numbers: 75.50.-y, 75.50.Lk, 75.50.Rr, 75.30.Kz

Recent years have seen an increased interest in properties of spin-glasses [1]. Particularly, a question concerning lower critical dimensionality has been addressed. Theoretical and numerical results agree that in thermodynamic limit there is no spin-glass phase (S-G) at finite temperatures in two-dimensional spin-glasses. In contrast, on the experimental time scales, the thin films appear to freeze (become irreversible) at a finite temperature, called $T_{\mathrm{f}}$, which is expected to decrease monotonically with the layer thickness $w$ [2].

Diluted magnetic semiconductors (DMS) have been known to exhibit fundamental spin-glass properties at low temperatures within a wide concentration range of the magnetic component. Recent progress in fabrication techniques, such as molecular beam epitaxy (MBE), has made it possible to investigate the properties of the S-G state of DMS in the form of very thin layers (down to a few ML) [3]. Therefore, a possibility now exists to investigate experimentally whether or not the S-G state indeed vanishes when the thickness of the Mn-rich layers is lowered to its critical value. A study of such an effect was initiated by Awschalom et al. [4]. They found from ac-modulated SQUID measurements that in (111)-oriented $\mathrm{CdTe} / \mathrm{Cd}_{0.80} \mathrm{Mn}_{0.20} \mathrm{Te}$ superlattices grown on $(100) \mathrm{GaAs}$, the spin-glass softened

\footnotetext{
${ }^{*}$ Work supported by the grant PBZ-Z011/P4/93/01 from the State Committee for Scientific Research (Republic of Poland).
} 
as the $\mathrm{Cd}_{0.80} \mathrm{Mn}_{0.20} \mathrm{Te}$ layer thickness was reduced to $12 \mathrm{ML}$ ( $1 \mathrm{ML}$ of CdMnTe $\simeq 3.2 \AA)$. For an even thinner layer $(w=6 \mathrm{ML})$ the $\mathrm{S}-\mathrm{G}$ was not observed at all. In our initial attempt to solve this problem [5] we found clear spin-glass fingerprints in superlattices (SLs) with either 24 or $6 \mathrm{ML}$ of $\mathrm{Cd}_{0.50} \mathrm{Mn}_{0.50} \mathrm{Te}$. On the other hand, we were then puzzled by the fact that only very faint traces of a phase transition were detected in the structure with $w=12 \mathrm{ML}$.

The above puzzling findings stimulated us to carry out detailed magnetic measurements on dedicated samples with improved quality. Namely, four CdTe/ $\mathrm{Cd}_{0.50} \mathrm{Mn}_{0.50}$ Te superlattices were studied [6]. In these SLs the thickness of the nonmagnetic CdTe interlayers was kept constant at $16 \mathrm{ML}(\simeq 52 \AA)$ while the thickness of the CdMnTe barriers varied from 20, through 15 and 10 down to $5 \mathrm{ML}$. The measurements of temperature dependent magnetic moment $m(T)$ were performed in a home-made, rf-biased SQUID magnetometer system equipped with a variable temperature insert. We studied the zero-field cooled (ZFC), the field cooled (FC), and the thermoremanent magnetization (TRM). The applied magnetic field was $H=1 \mathrm{kOe}$, and the dc magnetic susceptibility $\chi(T)$ was calculated from the relation $M(T)=\chi(T) H$, where $M(T)$ denotes the magnetization of the $\mathrm{Cd}_{1-x} \mathrm{Mn}_{x} \mathrm{Te}$ layers only.

We will first focus on the $\chi(T)$ results. As observed in other samples [5, 7], the four SLs exhibit greatly enhanced paramagnetic behaviour at the lowest temperatures, see Fig. 1a. Following suggestions based on the Zeeman splitting of excitons [8], and the suppression of exciton magnetic polaron [9], the effect is assigned to the presence of weakly interacting paramagnetic ions at non-abrupt $\mathrm{CdTe}-\mathrm{CdMnTe}$ interfaces, and size restrictions of large Mn clusters in the short-period SLs. In addition to studies with the magnetic field aligned in the plane of the layers [6], we present results of analogous measurements in the normal orientation. As seen in Fig. 1a, there is no orientation dependence within the experimental accuracy for any layer thickness. This finding, firstly, implies no noticeable magnetic anisotropy induced by the stress acting upon the magnetic layer embedded into the SL structure. Secondly, it seems that spatial restrictions in the studied structures on the Mn cluster size are not sufficient to produce any difference in the way the system responds to the orientation of the magnetic field.

Turning back to S-G properties, we underline that, contrary to the previously mentioned studies $[4,5]$, we clearly observe the basic fingerprints of the S-G state, i.e., a history dependent magnetization and TRM below $T_{\mathrm{f}}$, in all structures [6], see Fig. $1 b^{\dagger}$. This rules out the possibility for a discontinuous critical dependence of the S-G phase on $w$ in CdMnTe [4], at least in high- $x$ layers dominated by nearest-neighbour interactions and over the time scale probed in our experiments $\left(\sim 10^{2} \mathrm{~s}\right)$. Moreover, we see that on decreasing $w$, the transition to the S-G state ${ }^{\ddagger}$ shifts to a lower temperature. A similar effect has been reported

${ }^{\dagger}$ This result strongly suggests accidentally poor quality of the $12 \mathrm{ML}$ SL from the batch investigated in Ref. [5], particularly because the results for other two structures are generally in agreement with the SLs presently investigated.

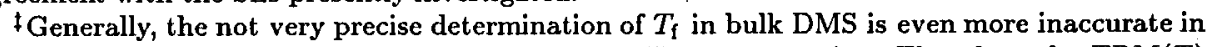
SLs, where the S-G cusp is obscured by "interfacial" paramagnetism. Therefore, the TRM $(T)$ dependence was used to determine $T_{\mathrm{f}}$ in SLs with much better accuracy. 

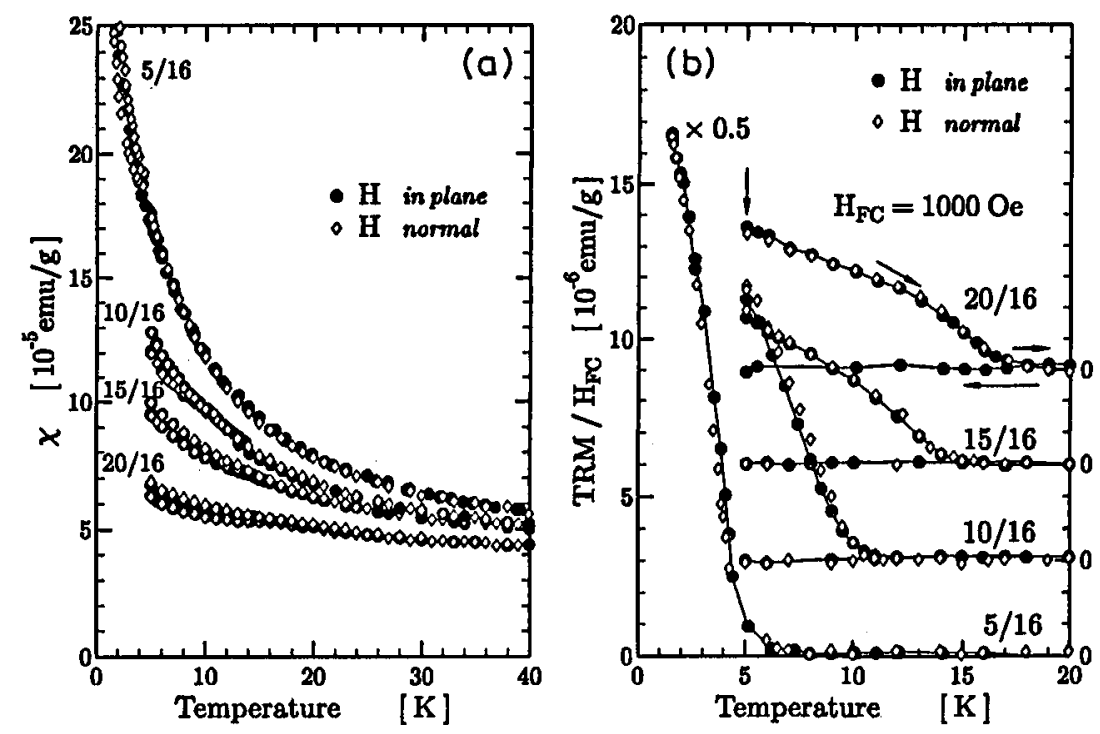

Fig. 1. Results of magnetic measurements for $\mathrm{Cd}_{0.50} \mathrm{Mn}_{0.50} \mathrm{Te}-\mathrm{CdTe}$ multilayers. The structures are labeled as $m / n$, where $m$ and $n$ are the number of magnetic/nonmagnetic monolayers respectively. The samples numbers are: 20/16: 06274A; 15/16: 06274B; 10/16: $06274 \mathrm{C}$; and 5/16: 06274D. The full symbols correspond to in plane orientation of the magnetic field, and the open symbols for the perpendicular configuration. (a) The temperature dependence of the dc magnetic susceptibility. The picture presents data for both ZFC and FC measurements for each orientation. (b) The temperature dependence of the thermoremanent magnetization. The data for different structures are shifted for clarity. The data were collected in the following way: (i) the samples were field cooled through $T_{\mathrm{f}}$ from the paramagnetic region, (ii) $H$ was removed at the lowest temperature ( 5 or $1.5 \mathrm{~K}$ ) and the moment (TRM) was measured on increasing temperature, (iii) at some high $T>T_{\mathrm{f}}$ the temperature sweep was reversed and additional points (above and below $T_{\mathrm{f}}$ ) were collected to establish the base line. Steps (ii) and (iii) are indicated by the arrows for 20/16 SL. The solid lines are guides for the eye only. Note the extremely high values of the TRM for the 5/16 multilayer.

for CdMnTe/CdMgTe structures [10]. Also, as shown in Figs. 1a and b, the properties of the $\mathrm{S}-\mathrm{G}$ do not depend on the orientation of the magnetic field. This is of particular interest, since it suggests that the critical thickness to affect dynamical processes below $T_{\mathrm{f}}$ is much smaller than $5 \mathrm{ML}$.

Now, let us focus on the dependence of $T_{\mathrm{f}}$ upon $w$, Fig. 2a. We find that $T_{\mathrm{f}}$ decreases monotonically with $w$, a theoretically expected result for thin layered spin-glasses measured over a long yet limited time. Since the expected relation has the form $T_{\mathrm{f}} \approx w^{a}$ [2], a double $\log$ plot of $\mathrm{T}_{\mathrm{f}}$ versus $w, \mathrm{Fig} .2 \mathrm{~b}$, allows us to estimate the value of the exponent $a$. Quite surprisingly, this value $(a \simeq 0.8 \pm 0.1)$ turns out to be very similar to that determined for CuMn and AgMn layers [11]. This finding may serve as yet another argument for using the term spin-glass for the 

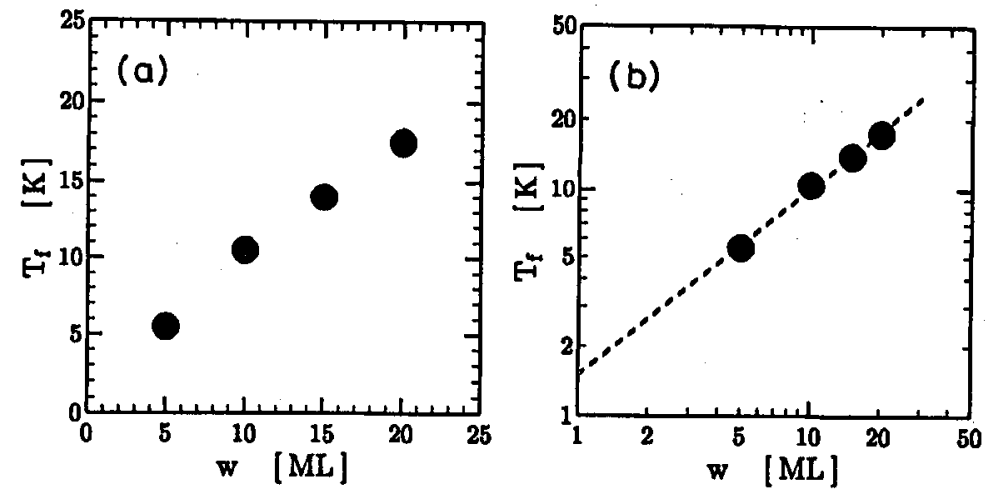

Fig. 2. Plots of $T_{\mathrm{f}}$ as a function of layer thickness. (a) Linear scale. (b) Double log scale. The dashed line represents a power law dependence of $T_{\mathrm{f}}$ on $w$ with $T_{\mathfrak{f}} \approx w^{0.8}$.

magnetic behaviour of DMS below $T_{\mathrm{f}}$. It seems that even though the microscopic mechanism that drives DMS into the S-G state is different from that in canonical spin-glasses, below $T_{\mathrm{f}}$ they are very much alike.

It is worth noting that extrapolation of the results gives a nonzero $T_{\mathrm{f}} \simeq 2 \mathrm{~K}$ for $w=1 \mathrm{ML}$. This is not totally misleading, since also the linear plot of $T_{\mathrm{f}}$ versus $w$ (Fig. 2a) does not show any discontinuity and suggests a nonzero $T_{\mathrm{f}}$ for $w=1 \mathrm{ML}$. This does not however tell us much about the lower critical dimensionality of our system as the final answer to this question is buried in the very large time domains of spin-glasses. Studies of the temperature dependence of the relaxation times are expected to clarify the situation [12].

In conclusion, SQUID studies of $\mathrm{Cd}_{1-x} \mathrm{Mn}_{x} \mathrm{Te}$ magnetic layers reveal the persistence of spin-glass irreversibilities down to $\simeq 16 \AA$ thin layers, i.e., well below the previously postulated $40 \AA$ for a quasi-2D threshold. The freezing temperature $T_{\mathrm{f}}$ is found to be a monotonic function of $w$ and obey a scaling law $T_{\mathrm{f}} \approx w^{a}$, with $a \simeq 0.8$, as found in canonical spin-glasses.

\section{Acknowledgment}

We are deeply indebted to Dr. M.A. Brummell for critical reading of the manuscript.

\section{References}

[1] See e.g., J. Bass, J.A. Cowen, in: Recent Progress in Random Magnets, Ed. D.H. Ryan, World Scientific, Singapore 1993 p. 177.

[2] D.A. Fisher, D. Huse, Phys. Rev. B 36, 8937 (1987).

[3] N. Samarth, H. Luo, J.K. Furdyna, T.M. Giebułtowicz, D.D. Awschalom, Acta. Phys. Pol. A 82, 573 (1992).

[4] D.D. Awschalom, J.M. Hong, L.L. Chang, G. Grinstein, Phys. Rev. Lett. 59, 1733 (1987). 
[5] M. Sawicki, S. Koleśnik, T. Wojtowicz, G. Karczewski, E. Janik, M. Kutrowski, A. Zakrzewski, E. Dynowska, T. Dietl, J. Kossut, Acta Phys. Pol. A 87, 169 (1995).

[6] M. Sawicki, S. Koleśnik, T. Wojtowicz, G. Karczewski, E. Janik, M. Kutrowski, A. Zakrzewski, T. Dietl, J. Kossut, Superlattices Microstruct. 15, 475 (1994).

[7] M. Sawicki, M.A. Brummell, P.A.J. de Groot, G.J. Tomka, D.E. Ashenford, B. Lunn, J. Cryst. Growth 138, 900 (1994).

[8] J.A. Gaj, W. Grieshaber, C. Bodin-Deshayes, J. Cibert, G. Feuillet, Y. Merle d'Aubigné, A. Wasiela, Phys. Rev. B 50, 5512 (1994).

[9] D.R. Yakovlev, G. Mackh, W. Ossau, A. Waag, G. Landwehr, R. Hellmann, E.O. Göbel, in: Proc. 22nd Int. Conf. on the Phys. of Semicon., Ed. D.J. Lockwood, World Scientific, Singapore 1995, p. 2613.

[10] M. Dahl, S. Gehrsitz, T. Litz, A. Waag, in: Proc. 22nd Int. Conf. on the Phys. of Semicon., Ed. D.J. Lockwood, World Scientific, Singapore 1995, p. 2593.

[11] L. Hoines, R. Stubi, R. Loloee, J.A. Cowen, J. Bass, Phys. Rev. Lett. 66, 1224 (1991).

[12] M. Sawicki et al., to be published. 